\title{
Desarrollo de competencias a través del uso de las herramientas Scratch y Arduino en niños y jóvenes pertenecientes a zonas urbanas marginales del Distrito Metropolitano de Quito
}

Children and youth skills development using Scratch and Arduino tools at marginal areas of the Metropolitan District of Quito

*FRANCISCO TERNEUS PÁEZ, JOHANNA TOBAR QUEVEDO, DAVID LOZA MATOVELLE, FERNANDO NARANJO HERRERA Universidad de las Fuerzas Armadas "ESPE",

Av. General Rumiñahui S/N y Paseo Escénico Santa Clara, Sangolquí, Ecuador,

*cfterneus@espe.edu.ec

\section{RESUMEN}

La búsqueda incansable para hallar una vinculación entre la tecnología y la educación responde a la necesidad diaria del progreso y crecimiento económico y social de un país. La educación es la formación destinada a desarrollar varias capacidades necesarias para la vida, la tecnología por su parte, se convierte en la herramienta para la adquisición de estos saberes de manera divertida, interactiva e innovadora, por esta razón la presente investigación enlaza estos dos aspectos. El objetivo principal es determinar las ventajas del uso de las plataformas Scratch y Arduino en niños y jóvenes pertenecientes a veinte Casas Somos del Distrito Metropolitano de Quito. La investigación presenta características descriptivas y emplea la técnica de observación para recolectar información y posterior análisis. Se contó con la participación de 380 niños entre 8 y 11 años de edad y 105 adolescentes entre 12 y 15 años. Obteniendo que el uso de la plataforma Scratch y Arduino permitió la potenciación del desarrollo cognitivo, razonamiento lógico, operaciones lógicas del pensamiento y ubicación espacial, de los participantes, quienes propusieron como resultado la programación y diseño de diferentes proyectos enmarcados en la resolución de un problema de la vida cotidiana.

Palabras clave:

Scratch, Arduino, desarrollo de competencias, conocimiento tecnológico, barrio urbano - marginales. 


\section{ABSTRACT}

The tireless search to find a link between technology and education responds to the daily need for progress and the economic and social growth of a country. Education is the training aimed at developing various skills necessary for life, technology for its part, becomes the tool for acquiring this knowledge in a fun, interactive and innovative way, for this reason, the present research linked to these two aspects. The main objective is to determine the advantages of using the Scratch and Arduino platform in children and youth belonging to twenty Casas Somos of the Metropolitan District of Quito. The research has descriptive characteristics and uses the observation technique to collect information and subsequent analysis. There were 380 children between 8 and 11 years old and 105 teenagers between 12 and 15 years old. Obtaining the use of the Scratch and Arduino platform programmed the potentialization of cognitive development, logical reasoning, logical operations of thought and spatial location, of the participants, who proposed as a result the programming and design of different projects framed in the resolution of a problem of everyday life.

Keywords: Scratch, Arduino, skills development, technological knowledge, urban - marginal neighborhood.

\section{INTRODUCCIÓN}

La academia busca soluciones para promover la inclusión económica, promoción social y supresión progresiva de la pobreza; enfocándose en zonas vulnerables, especialmente en las que forman parte del programa erradicación del trabajo infantil. Los conocimientos tecnológicos son considerados ejes fundamentales para el desarrollo social y económico, por cuanto surge la importancia de brindar oportunidades que permitan acceder al conocimiento mediante el acceso a Internet y equipamientos tecnológicos en zonas urbano marginales.
La programación y elaboración de este tipo de productos destinados al aprendizaje, fortalece la construcción del razonamiento analítico y resolución de problemas, mismos que son indispensables para el desenvolvimiento cotidiano. Por cuanto denota necesario afianzar competencias intelectuales y sociales, a través de actividades interactivas que respondan a las exigencias de la sociedad de la tecnología e información (Blanco, 2009). Es por ello que, la Universidad de las Fuerzas Armadas ESPE, se propuso formar vínculos y alianzas estratégicas con diferentes instituciones gubernamentales y no gubernamentales, para que plataformas de programación puedan llegar a la infancia y adolescencia en sectores vulnerables del país con el objetivo de un crecimiento social.

El proyecto de vunculación se incursionó en espacios de desarrollo social integral creados por el Municipio de Quito denominados Casas Somos, en estos sitios se pretende potencializar capacidades y competencias intelectuales, artísticas y sociales, teniendo un enfoque de inclusión y equidad, para ello disponen de talleres de formación ciudadana, culturales, artísticos, cursos de computación, apoyo escolar, actividades lúdicas, deportivas y culturales destinados a niños, jóvenes, adultos y adultos mayores, los cuales son accesibles dentro de los barrios y diferentes sectores de la ciudad.

Al momento existen cuarenta y dos Casas Somos distribuidas en las distintas administraciones zonales (La Hora, 2017) (Municipio del Distrito Metropolitano de Quito, 2018), sin embargo se ha realizado el acercamiento únicamente con las comunidades: Alangasí, Amaguaña, Argelia, Calderón, Carapungo, Carcelén alto, Carcelén bajo, Guangopolo, Guápulo, IESS-FUT, La Roldós, La Tola II, Lloa, San Bartolo, San José de Monjas, San Marcos, Toctiuco, Zámbiza, Tumbaco y Atahualpa; todas ellas pertenecientes a la ciudad de Quito. Se aplicaron 23 talleres de capacitación con la temática en Scratch y Arduino, permitiendo la participación de 380 niños y 105 adolescentes,

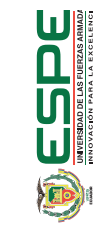


quienes tuvieron la capacidad de comprender la tecnología y sus usos a través de aplicaciones tangibles y prácticas, direccionados a una visión de mejora o solución a problemas cotidianos.

Scratch ayuda a los usuarios a realizar sus proyectos personalmente atractivos, motivadores y significativos, además facilita acciones como importar o crear varios tipos de medios tales como; imágenes, sonidos y música. El sitio Web de la mencionada herramienta digital proporciona un contexto social para sus usuarios, permitiendo compartir proyectos, recibir comentarios y brindar retroalimentación a otros usuarios en base a proyectos ya desarrollados, con la finalidad de proveer información a aquellas personas que no tienen una previa experiencia en el sitio Web.

Para dar paso a la ejecución del proyecto de Vinculación con la Sociedad, el Departamento de Ciencias de la Energía y Mecánica de la Universidad de las Fuerzas Armadas ESPE, emprendió las acciones necesarias para organizar los módulos y metodología de estudio que van a seguir los estudiantes universitarios de las carreras de ingeniería mecánica y mecatrónica, quienes gustosos compartieron sus conocimientos en programación, se vincularon directamente con la población, desarrollaron habilidades y competencias en manejo de grupos, experiencia técnica y desenvolvimiento en público, con la finalidad de determinar las ventajas del uso de la plataforma Scratch y Arduino en niños y jóvenes pertenecientes a veinte Casas Somos del Distrito Metropolitano de Quito. para emitir una respuesta oportuna. Ante esta situación se realizan dos procesos complemetarios; el primero que se refiere a la incorporación de información sobre esquemas preexistentes (asimilación); y el segundo proceso que se encarga de modificar esos esquemas para acomodar la nueva información (acomodación).

Ahora bien, estudios demuestran que las edades enmarcadas entre los 0 a 6 años son trascendentales en la vida del ser humano, debido a la gran cantidad de neuronas cerebrales que realizan conexiones sinápticas, las cuales al recibir estimulación adecuada permiten formar aprendizajes duraderos y un mayor desarrollo de las operaciones lógicas del pensamiento, dentro de un contexto social y afectivo (Tinajero, 2014).

Por otro lado, la plasticidad cerebral no es más que crear nuevas conexiones cerebrales aun cuando no haya habido lesiones, esto se logra mediante el entrenamiento cerebral basados en actividades lúdicas que representen desafíos cognitivos y motrices a ser superados (Rosenzweig \& Bennet, 1996). Es decir, el valor pedagógico del juego está presente en la mejora de habilidades sociales, desarrollo operaciones mentales de orden superior, interacción intra e interpersonales (Ojeda, Carpio, 2015).

La lúdica o juego está presente en todas las etapas evolutivas del ser humano, ya que, permite el desarrollo psicosocial, adquisición de saberes, formación de la personalidad, enmarcadas dentro de actividades que permiten el placer, conocimientos, actividad creativa y disfrute (Ojeda, Carpio, 2015); empero existen otros beneficios a los cuales responde, por ejemplo: Desenvuelve la concentración y agilidad mental, aumenta la circulación sanguínea, libera endorfina y serotonina, proporciona la inclusión social, aumento de la autoestima, desarrollo de la creatividad y pensamiento, estimulación de la socialización, preparar al niño al mundo laboral, entre otras.

Al conocer los beneficios del juego, se puede comprender su aplicabilidad en el desarrollo cognitivo; por consiguiente es necesario 
identificar las habilidades que son necesarias para el desarrollo del pensamiento lógico-matemático, el cual resulta imprescindible durante las etapas iniciales, debido a que permite comprender, entender, organizar y desenvolverse en la realidad a través del razonamiento analítico.

En investigaciones realizadas se menciona que para la construcción del pensamiento lógico matemático es necesario desarrollar las siguientes capacidades en el niño (Rubio, Centeno, 2012):

- La observación: va más allá de la acción de mirar, debido a que permite un análisis de características que contiene un estímulo, al cual responde a través de la atención voluntaria. Esta capacidad se puede potencializar a través de juegos que beneficien a la percepción de propiedades y relaciones entre las mismas.

- La imaginación: se la puede interpretar a través de una acción creativa, la cual permite la modificación y mejora de estructuras establecidas. Dentro del aprendizaje matemático se puede evidenciar en la capacidad de adaptar dichos conocimientos en diferentes situaciones para resolver problemas.

- La intuición: a través de la misma se puede encontrar la verdad sin la necesidad de un razonamiento profundo; sin embargo, al desarrollarla permite que el niño distinga lo real de lo ficticio mediante sus pensamientos que conoce y valida como racionales y verdades.

- El razonamiento lógico: razonar es la forma en la que el pensamiento parte de una o varias verdades, como premisas con la cuales se llegan a conclusiones que responden a reglas de inferencia.

\section{Pensamiento lógico}

El niño abandona un pensamiento concreto basado en hechos y realidades visibles, el mismo que es funcional a través de la interpretación y conceptos básicos que construye en edades iniciales. Ingresando a un mundo abstracto y racional a través de pensamientos y conceptos, razonamientos superiores, operaciones lógicas del pensamiento y por tanto desarrolla un pensamiento lógico que beneficia sus decisiones cotidianas.

Uno de los objetivos que busca la tecnología es mejorar la calidad de vida de las personas, sin embargo, para lograrlo se requiere llegar a una invención. Para ello la persona sigue un proceso creativo, cuyos elementos no responden a una continuidad cronológica, ya que, se consideran de manera integral; se podría traducir como un caleidoscopio creativo hasta llegar a la invención (Hernández, Londoño, 2016), como indica la Fig. 1.
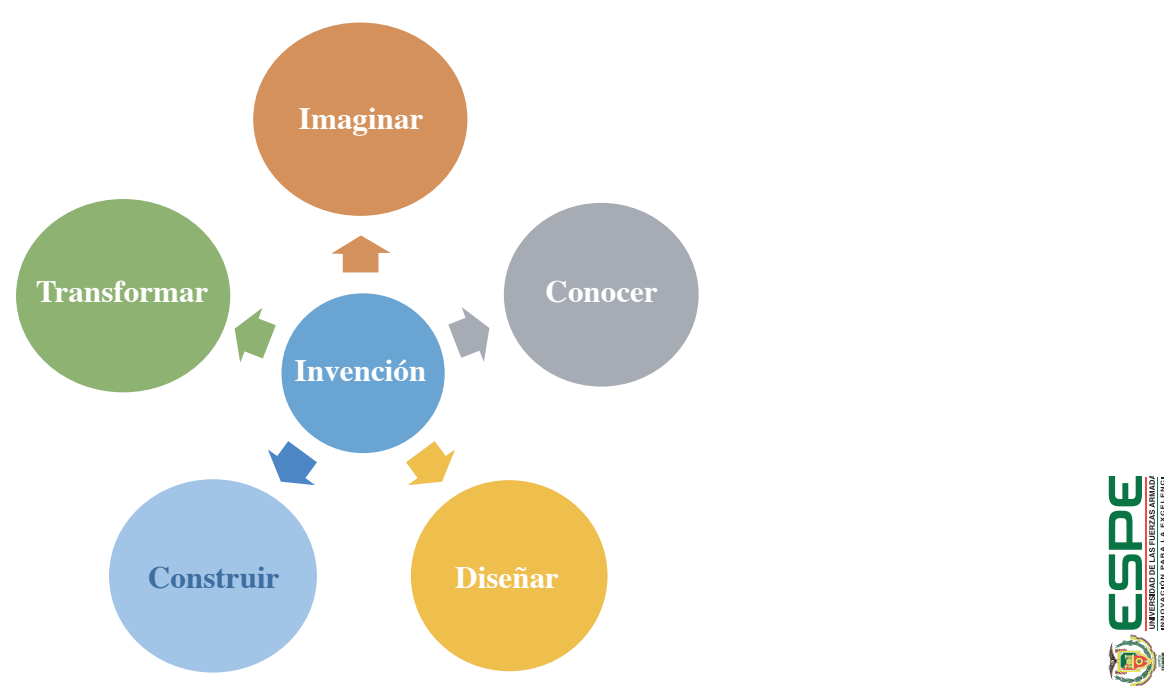

Fig. 1 Proceso creativo complejo de la invención. 
Una de las bases que ayudan a conseguir la invención es la robótica, la cual será a futuro una fuente generadora de empleos, originados por estas mismas tecnologías (Jiménez, 2015).

Debido a su poder creador, como oportunidades dentro de la misma industria para generar a través de la creatividad nuevas soluciones a posibles necesidades. De hecho, en la actualidad la humanidad vive rodeada de equipos que tienen funciones autónomas y colaboran con el ser humano, principalmente en la industria manufacturera realizando funciones repetitivas o peligrosas; pero también en servicios profesionales como inspección, rescate, limpieza; y también personales y domésticos como de seguridad en vivienda, para tareas dentro del hogar, entretenimiento, etc. Por consiguiente, en el futuro cercano, se esperan grandes avances en los ámbitos de salud, la educación y domiciliares.

De esta manera, la programación y robótica serán consolidadas como un medio de producción y desarrollo. En este punto radica la importancia de adaptación a nuevas tecnologías desde la primera infancia, consolidando este progreso a través de actividades interactivas que permitan el acercamiendo y el aprendizaje de las mismas.

\section{Plataformas accesibles}

Las plataformas Scratch y Arduino, cumplen con el objetivo de captar la atención e interés de los usuarios, provocando reacciones de emoción durante la realización de pequeñas actividades de programación y al evidenciar sus resultados. La plataforma ayuda al desarrollo del pensamiento lógico, fomenta la creatividad, mejora la habilidad de comprensión, fortalece la atención focalizada, entre otros (Arribas, 2016).

Los niños tuvieron la oportunidad de interactuar y experimentar con herramientas y materiales electrónicos específicos logrando armar pequeños robots a quienes se les dio movimiento en base a condiciones de sensores. Pues, aprendieron a reconocer los materiales, identificar los puertos del hardware y realizar conexiones básicas. Esta interacción que mezcla la intuición con la teoría son el sustento que Hong \& Trepanier-Street (2004 . p. 90) asegura que: "La experimentación habilita y mejora las condiciones de aprendizaje, debido a que los niños procuran actuar y construir de manera individual e independiente."

Por otro lado, los términos de cibercultura y educación, permiten entender cómo a través de la educación es posible generar mejores gestiones de conocimiento para quienes manejan y accedan a plataformas tecnológicas (llvay, Taday, 2014). Recalcando que los beneficios serán a nivel económico, cultural y social, debido a que posibilita la adquisición de trabajos futuristas y soluciones a problemáticas sociales. Algunas de las características que requieren los software educativos, son: ser flexibles y adaptables para diversos usuarios, que fomenten una comunicación interpersonal; y, que reciban retroalimentación. Es por esta razón que para la aplicación de los talleres en Casas Somos se tomaron en cuenta las siguientes plataformas:

\subsection{Scratch.}

Se trata de un lenguaje de programación visual desarrollado por el MIT Media Lab, el cual se encuentra dirigido a cualquier grupo poblacional, entre ellos: estudiantes, académicos, profesores y padres de familia, que deseen incursionarse a crear animaciones, juegos e interacciones (Marii, 2014). Por otro lado, los componentes visuales que provee el programa permite desarrollar pequeños juegos, gráficas animadas, historias interactivas, entre otros, con la finalidad de lograr que los docentes o adultos responsables inicien o fortalezcan conceptos básicos propios a edades tempranas. 
Scratch posee una interfaz de usuario amigable, debido a que conserva diversas formas de crear fondos y objetos personalizados, a los cuales se accede desde la biblioteca o se los puede hacer con el propio editor de Scratch, al mismo que se le puede asociar instrucciones mediante bloques como: movimientos, apariencia, sonido, lápiz, datos, eventos, control, sensores, operadores (Bailey, 2018). En definitiva, su uso permite desarrollar el conocimiento de manera completa, lúdica y apegándose a las exigencias de la era tecnológica.

\subsubsection{Estilo de Programación.}

Para muchos autores, la programación de computadores vuelve tangibles las matemáticas y la computación mediante procesos de diseño en los que los estudiantes se comprometen en la solución de problemas de manera experimental y repetitiva (Bourret). Scratch ofrece una interfaz intuitiva y muy fácil de comprender, permite manipular imágenes, fotos, sonido, música, etc. y sobre todo, una forma de programación visual con todos estos elementos. Scratch está disponible sobre Windows, Mac OS $X$ y Linux y se difunde bajo licencia libre MIT.

Aprender a programar tiene que ver, en el fondo, con aprender a pensar lógicamente y aprender a abordar los problemas metódicamente. Ciertamente, es difícil iniciarse en la programación con los lenguajes tradicionales como Java o $\mathrm{C}++$. Al trabajar con Scratch se comprenden fácilmente conceptos matemáticos e informáticos que están muy bien integrados en el programa, como son: Los procesos interactivos, los criterios condicionales, las coordenadas en un plano, las variables, etc.

Los bloques de construcción sobre los que se apoyan los programadores para construir soluciones son relativamente simples: secuencia, iteración y condición. En Scratch, esos bloques ya están implementados y para utilizarlos sólo es necesario arrastrarlos al programa que se quiere construir. Además, los bloques de código se pueden cambiar de forma dinámica y el resultado se observa inmediatamente, como se puede observar en la Figura. 2.

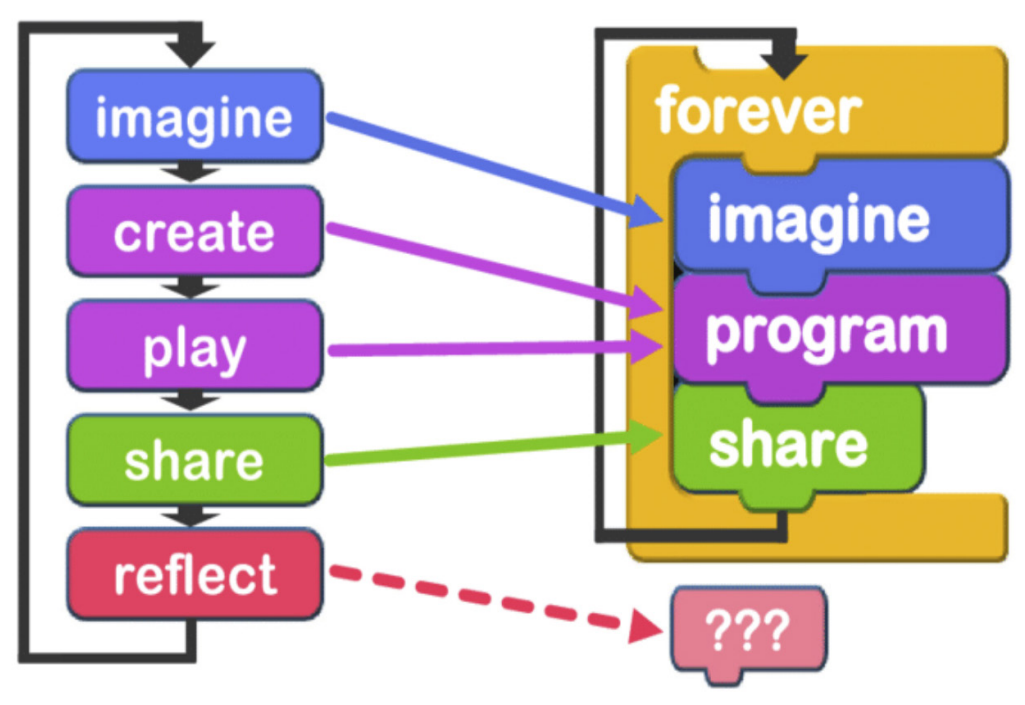

Fig. 2 Procesos en plataforma Scratch

Scratch permite enfrentarse a la resolución de problemas rápidamente, sin necesidad de conocer la sintaxis, a diferencia de los lenguajes de programación tradicionales con los que la curva de aprendizaje es plana, ya que es necesario aprender a leerlo y escribirlo antes de ponerse a trabajar con ellos. Estos procesos de concepción de proyectos y su construcción, desarrollan las competencias necesarias para llegar a tener un pensamiento creativo, una comunicación clara, un análisis sistemático, capacidad de colaboración, la reflexión interactiva, etc. 


\subsubsection{Elementos de la interfaz.}

La interfaz de usuario de Scratch se esfuerza por facilitar la navegación. Utiliza una ventana única, diseño de paneles múltiples para garantizar que los componentes clave siempre estén visibles. Tiene cuatro paneles principales, en la parte izquierda se encuentra la paleta de comandos con botones para seleccionar categorías. Los objetos que realizan acciones en un proyecto se denominan sprite y la mayoría los tienen debido a que son los únicos que tienen la capacidad de movilizarse.

Las partes de la interfaz se describen en la Figura. 3.

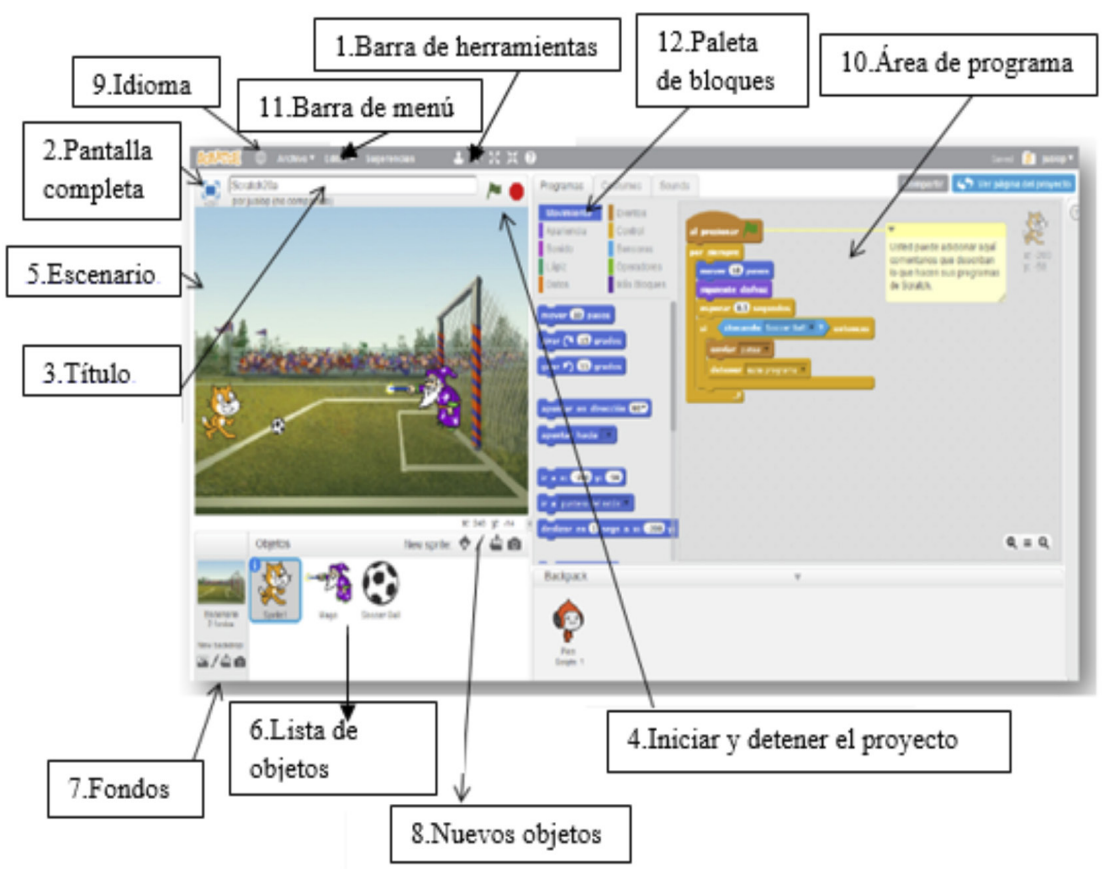

Fig. 3 Partes de interfaz de Scratch

\subsection{Arduino}

Es una plataforma de prototipos electrónicos de código abierto. Es decir, posee recursos que se pueden usar, redistribuir o reescribir de forma gratuita, a menudo software o hardware. A través de tecnología que hace uso del movimiento controlado de señales eléctricas. Mediante una arquitectura de hardware en la que un software puede ejecutar. Arduino es una herramienta para hacer que los ordenadores puedan sentir y controlar el mundo físico a través de un ordenador personal.

Es una plataforma de desarrollo de computación física denominada en inglés como physical computing, esta es de código abierto, basada en una placa con un sencillo microcontrolador y un entorno de desarrollo para crear software para la placa.

El microcontrolador es una serie de circuitos integrados en los que se puede grabar las instrucciones en lenguaje de programación para crear programas que interactúen con los circuitos de la placa.

Por lo tanto, este microcontrolador posee una interfaz de entrada donde se puede conectar diferentes tipos de periféricos que permitan enviar datos (imágenes, datos, diferentes tipos de sensores) al microcontrolador, así mismo cuenta con un interfaz de salida para reproducir los datos procesados.

En definitiva, la placa Arduino es como una pequeña computadora que puede ser programada tantas veces como sea necesario. Como una computadora, proporciona interacción de E / S, a través de entradas y salidas digitales y pines de entrada analógica. La figura 4 detalla sus componentes. 


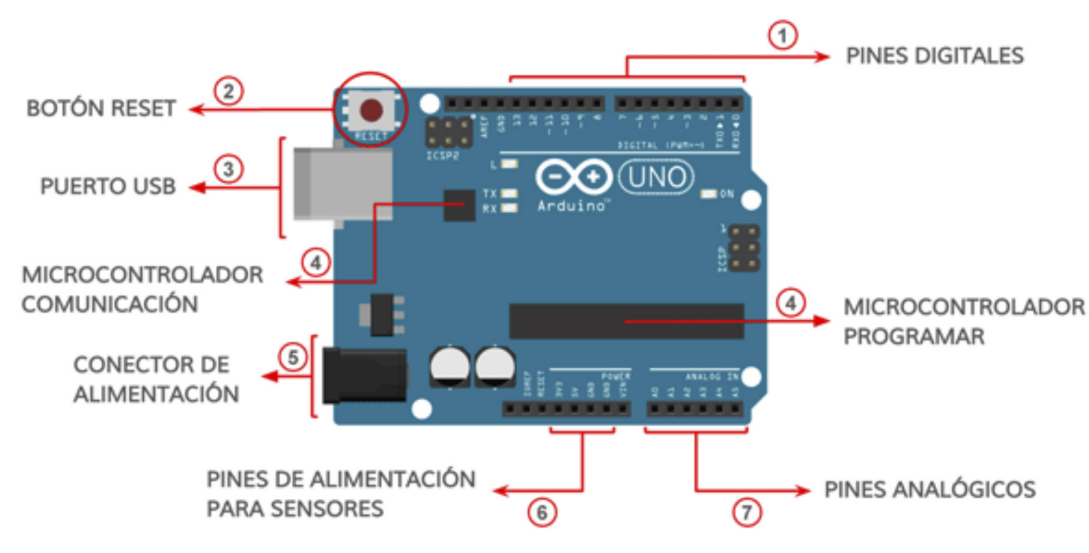

Fig. 4 Placa Arduino 1

\subsection{Scratch para Arduino.}

Es una modificación de Scratch que permite programar de manera interactiva en la plataforma y comunicar con la tarjeta de hardware libre Arduino de forma sencilla, proporcionando una interfaz amigable para niños que inician en el área tecnológica con funciones básicas como encender un diodo led desde el computador, hasta la programación de pequeños robots controlados por mando a distancia. En otras palabras, Scratch permite una programación simple mediante bloques con figuras geométricas específicas que permiten emparejar unas con otras, las cuales no permiten al usuario cometer errores durante la lógica de programación al intentar conectar funciones no permitidas entre sí.

Las tarjetas compatibles y recomendadas para la programación en S4A e interacción física son las placas Arduino Uno, Demilanove y Diecimila, las cuales tienen características muy similares entre sí en cuento a puertos analógicos y digitales, sin embargo por poseer una mejor arquitectura el primero de ellos es el más usado.

Para el protocolo de comunicación entre el programa S4A y la placa Arduino es necesario un firmware específico (PicoBoard) que permita la correcta transmisión de datos, el tiempo de respuesta, es decir, el tiempo que se realice una acción en el programa y evidenciar la reacción en la tarjeta es de 75 milisegundos, este tiempo de respuesta es bidireccional en cuanto a comunicación.

Dentro de la conectividad de la tarjeta se debe tener en cuenta que el programa tiene pines específicos para ciertos actuadores como los servomotores que tienen asignados los pines digitales $(4,7,8$ y 12) para el Arduino uno, para una transmisión de datos de forma inalámbrica se permiten dispositivos bluetooth (HC-06) y wireles (Xbee).

\section{MÉTODO}

El estudio es de tipo descriptivo, buscando especificar las propiedades, las características y los perfiles de personas, grupos, comunidades, procesos, objetos o cualquier otro fenómeno que se someta a un análisis, (como dice Hernández, Fernández \& Baptista 2014). Es decir el análisis de las ventajas que podría generar el uso de las plataformas Scratch y Arduino en niños y jóvenes de Casas Somos.

Por otro lado, se emplea la técnica de observación no participante, es decir cuando el investigador no se pone en contacto personalmente con el hecho o fenómeno que trata de investigar, por el contrario, la información la toma de forma externa. 


\section{Participantes}

El estudio se realizó bajo una muestra probabilística, la población consignada para dicho procedimiento es de 380 niños entre 8 y 11 años de edad y 105 adolescentes entre 12 y 15 años, pertenecientes a veinte Casas Somos del Distrito Metropolitano de Quito; Alangasí, Amaguaña, Argelia, Calderón, Carapungo, Carcelén alto, Carcelén bajo, Guangopolo, Guápulo, IESS-FUT, La Roldós, La Tola II, Lloa, San Bartolo, San José de Monjas, San Marcos, Toctiuco, Zámbiza, Tumbaco y Atahualpa; todas ellas pertenecientes a la ciudad de Quito.

\section{Instrumento}

Los instrumentos empleados fueron encuestas y entrevistas:

El primero fue dirigido a cuatro coordinadores Casas Somos, un docente de la Universidad de las Fuerzas Armadas ESPE, un representante de Fundación Telefónica Ecuador y un representante de Conquito.

El cuestionario constó de 10 preguntas, mismas que ofrecieron su puntuación mediante la escala de Liker, misma que tiene como mínima puntuación pésima y máxima como excelente. A continuación, se muestra el cuestionario:

2. ¿̇Fue usado de manera apropiada los materiales para facilitar el aprendizaje?

¿Considera que el curso impartido fue adecuado para niños?
3. ¿Cuán útil fue el curso para los niños en su vida estudiantil?

4. ¿ ¿Cuál fue el interés de los niños acerca del curso?

5. ¿Cuánto ayudo a mejorar la concentración de los niños?

6. ¿Cuál fue la satisfacción general del curso?

7. ¿Cuánto incremento de participación de los niños?

8. ¿Cuánto aumento el interés de los niños por carreras técnicas?

9. ¿Considera que los instructores supieron transmitir conocimiento?

10. ¿Considera que se debe realizar más cursos de este tipo a futuro?

En cuanto a las entrevistas, se desarrollaron en base al primer instrumento, donde se recolectó opiniones, recomendaciones, datos objetivos y observaciones de los organizadores y de los coordinadores de las casas Somos que son quienes tienen contacto directo con los beneficiarios. El cuestionario constó de 7 preguntas, las cuales se muestran a continuación:

1. ¿̇A qué tipo de población fue dirigido el curso?

2. ¿ ¿Cuál es su opinión del curso?

3. ¿Cómo benefició estos cursos a los niños que lo recibieron?

4. ¿ Cree usted que los resultados obtenidos en el curso generaron alguna forma de cambio de vida en los niños? 
5. ¿Cree que estos cursos fomenten a los niños a seguir carreras técnicas?

6. ¿Cree usted que se deben realizar más actividades como la de los cursos de Arduino-Scratch?

7. ¿Cuál cree usted que fue el impacto generado por el proyecto?

\section{RESULTADOS}

En el caso de los niños y jóvenes de los talleres de capacitación con la temática en Scratch y Arduino en las Casas Somos, se obtuvo como resultado el involucramiento de los niños y jóvenes en la programación en Scratch, quienes dieron rienda suelta a su imaginación, elaboraron sus propios videojuegos, utilizando sentencias propias de programación, como bucles, condicionales, plano cartesiano, disfraces y otros, como se muestra en la Figura 5.

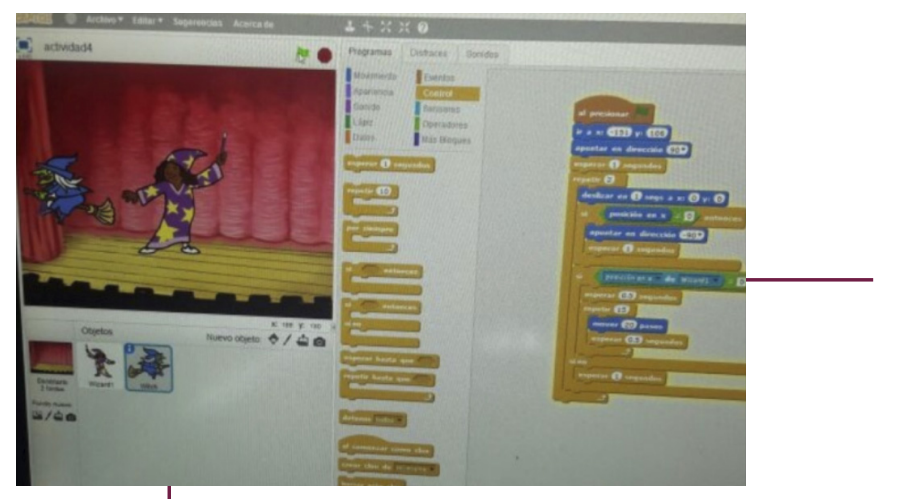

Fig. 5 Ejemplos de programas desarrollados con Scratch
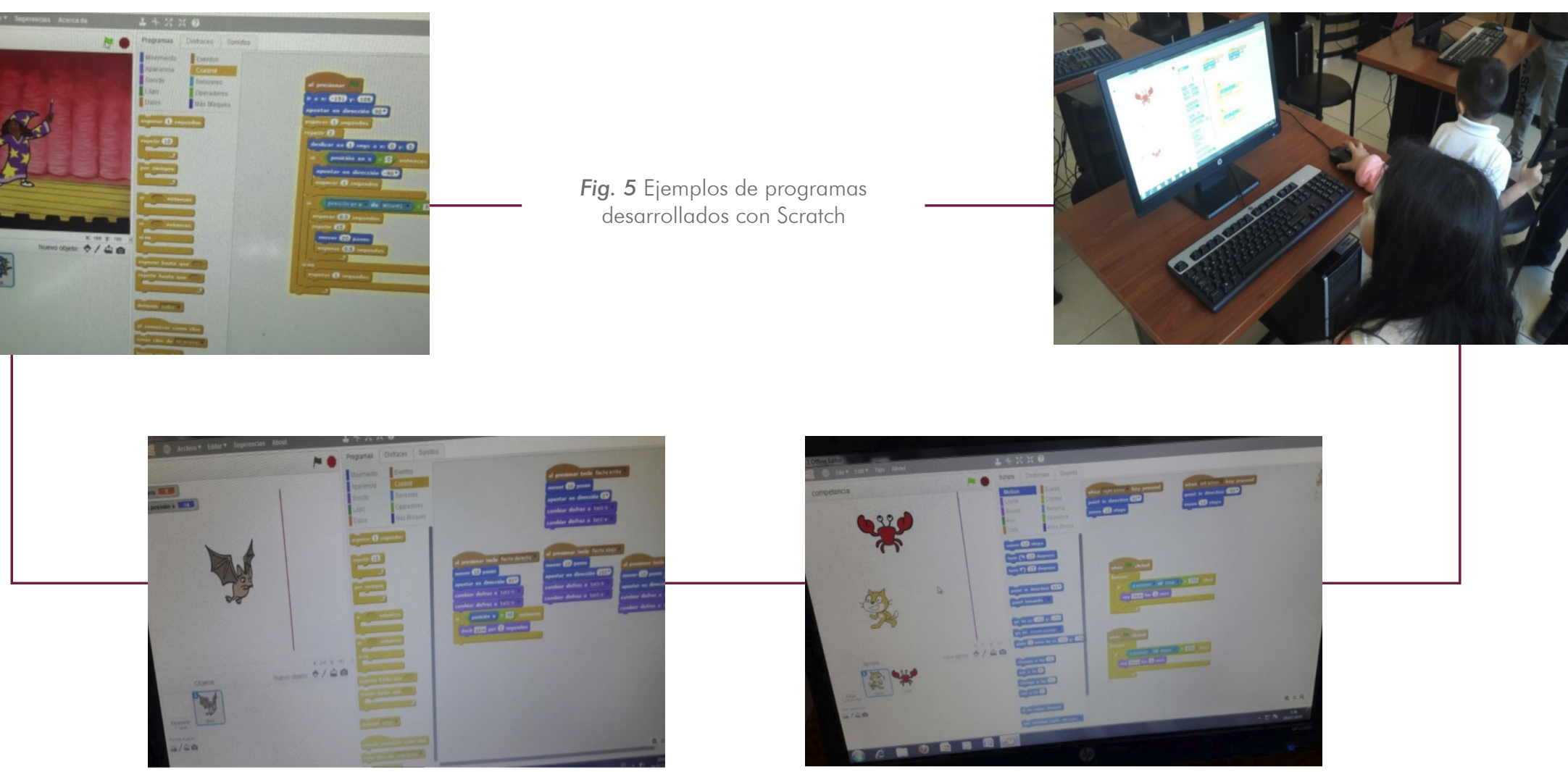
Desarrollo de competencias y habilidades en jóvenes y niños a través del uso de las herramientas Scratch y Arduino

Francisco Terneus P., Johanna Tobar Q., David Loza M., Fernando Naranjo H. • VínCCULOS-ESPE (2019) VOL. 4, No.2:30-44

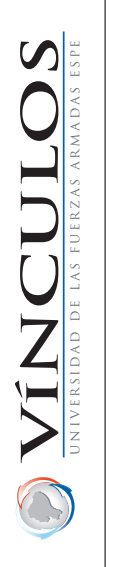

U!l!

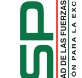

山l|

亩:

En el caso de Arduino se crearon pequeños juegos interactivos que prendían las luces en diferentes secuencias, o ante diferentes estímulos ya sea temporales, sonoros o lumínicos; o la construcción de pequeños vehículos donde hay un motor para cada rueda, lo que permite subir obstáculos y curvar; o una pequeña grúa que permite cerrar la pinza ante la presencia de un objeto, esto se muestra en la Figura. 6
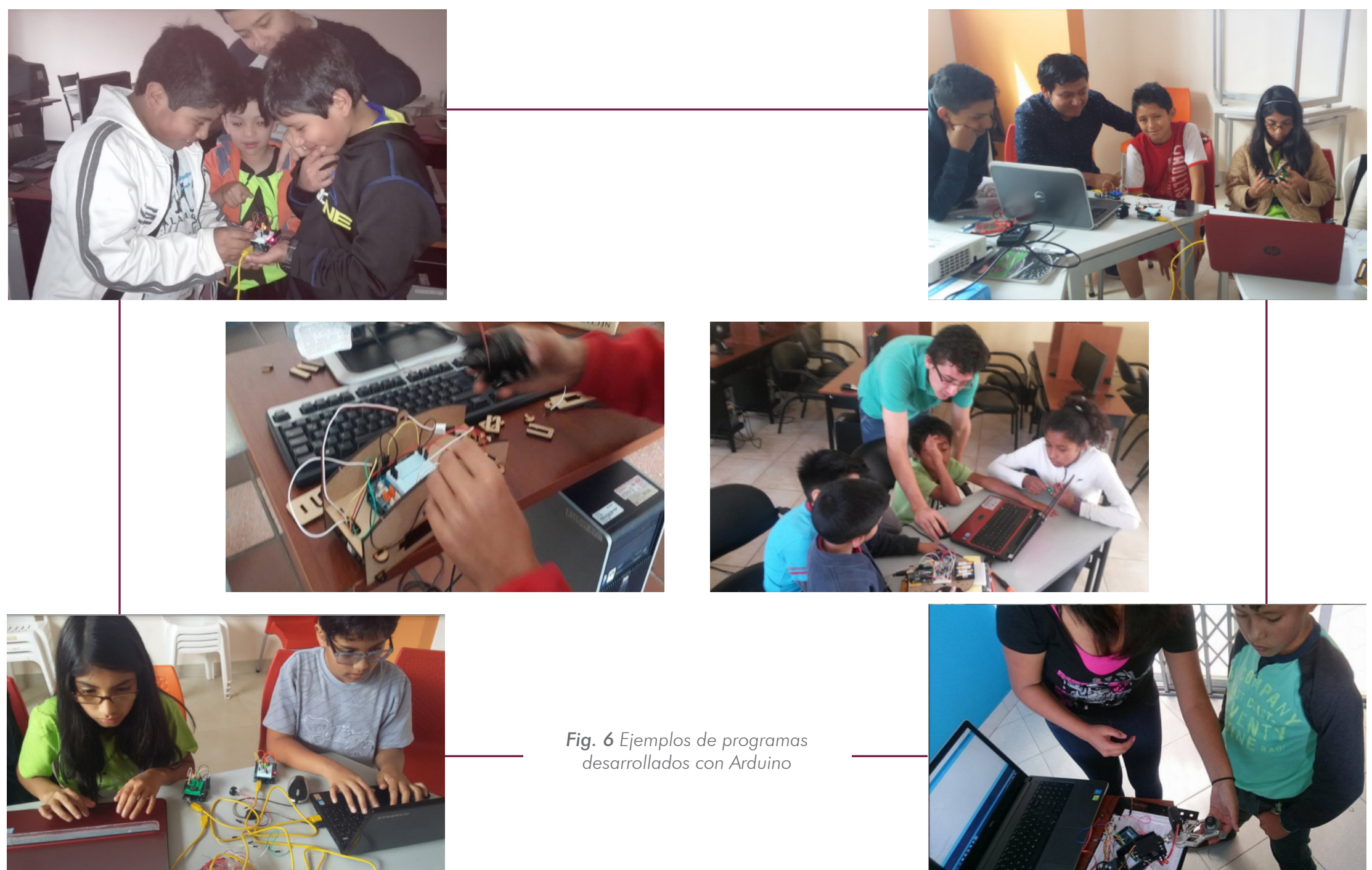

Fig. 6 Ejemplos de programas desarrollados con Arduino
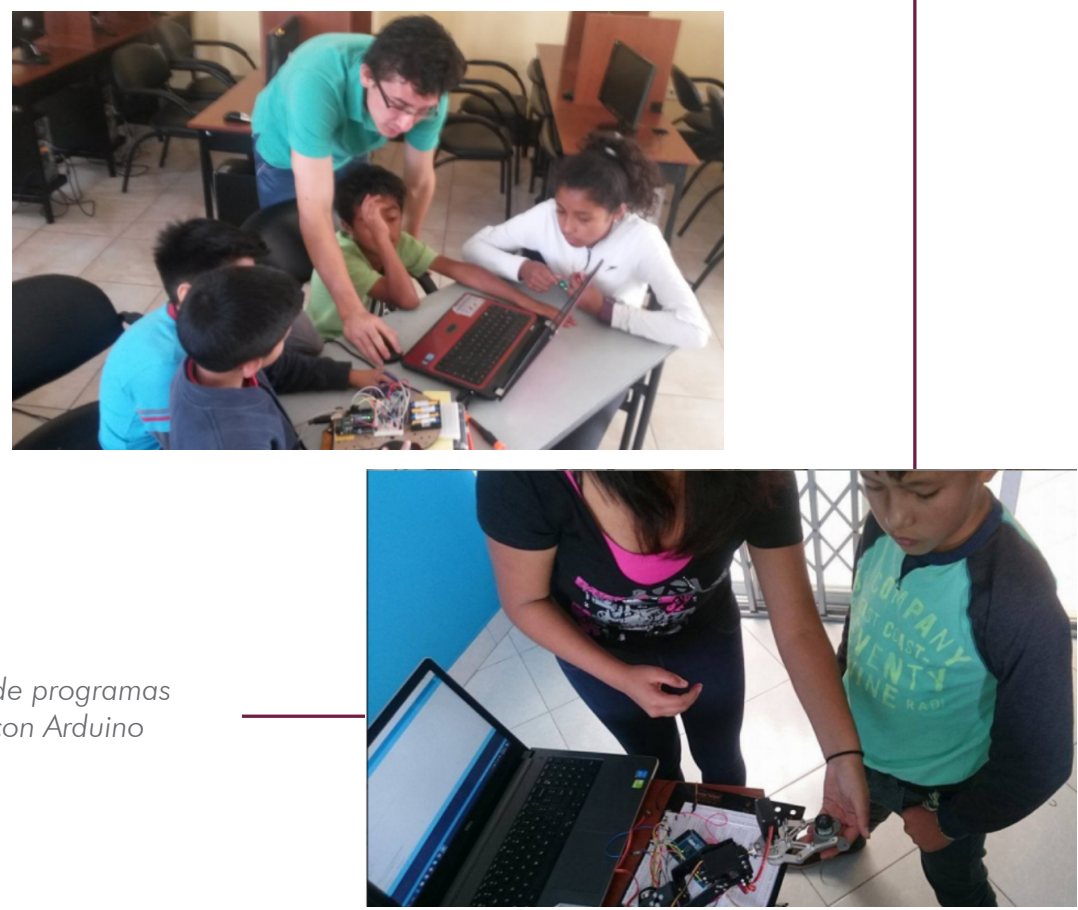


\section{DISCUSIÓN Y CONCLUSIÓN}

Una vez comprendidas las particularidades que hicieron este proyecto posible, es importante reconocer los resultados obtenidos después de la aplicación, los cuales permiten responder a la necesidad inicialmente planteada y reconocer el aporte de la Universidad de las Fuerzas Armadas ESPE al crecimiento social.

Al término del proyecto, se realizó la medición de impacto del proyecto a los coordinadores de Casas Somos a través de la aplicación de entrevistas y encuestas, logrando resaltar los siguientes resultados:

La respuesta a la pregunta de: ¿̇Cuán útil fue el curso para los niños en su vida estudiantil?, las respuestas resultaron en que el 85,7\% de la población encuestada consideró que la influencia del curso ha sido útil en el ámbito académico de los niños participantes, como indica la Figura. 7

\section{¿Cuán útil fue el curso para los niños en su vida estudiantil?}

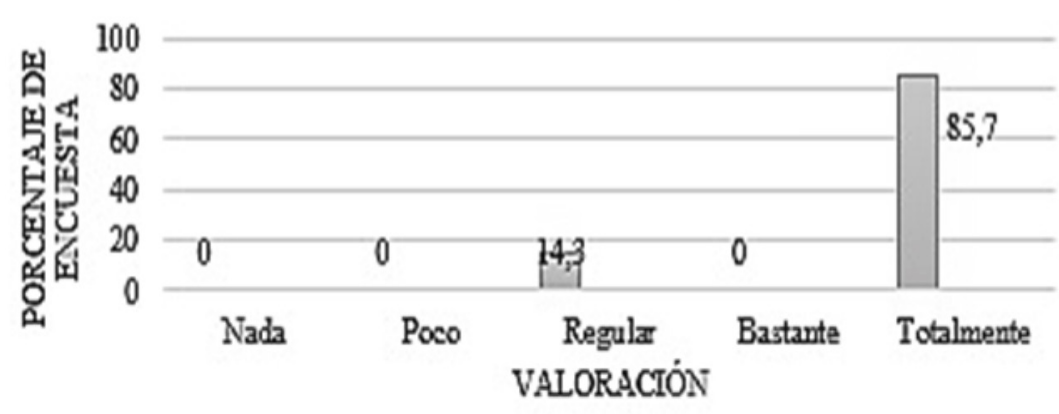

Fig. 7 Porcentaje de utilidad del curso en la vida estudiantil.
Teniendo en cuenta que para mejorar dicho ámbito es necesario desarrollar habilidades cognitivas como; clasificación, seriación y correspondencia, para la construcción de un pensamiento lógico. Todo esto fue trabajado a lo largo de la realización de los proyectos, debido a que la programación requiere de seleccionar las variables correctas y ubicarlas en un orden lógico para su correcto funcionamiento, logrando que el programa fuera útil dentro de la vida estudiantil.

Por otro lado, se consideró a la concentración como variable a ser estudiada, destacándose como la capacidad cognitiva básica para el desarrollo de las operaciones lógicas del pensamiento. A la pregunta: ¿Cuánto ayudó para mejorar la concentración en los niños? , se pudo determinar que el $42,8 \%$ de los coordinadores consideró que dicha capacidad aumentó totalmente en los niños y jóvenes participantes, como indica la Figura. 8. Siendo un resultado representativo, ya que, a partir de la concentración surge la posibilidad de desarrollar, crear y plasmar nuevas ideas en los productos terminados a través de un proceso creativo que finaliza en la innovación, el cual afianza sus capacidades cognitivas.

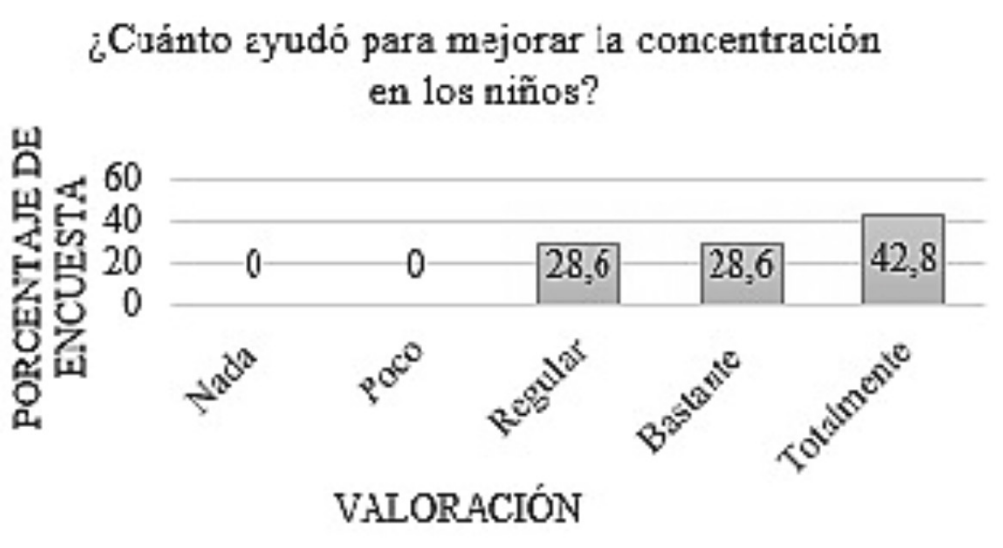

Fig. 8 Porcentaje de aumento de concentración

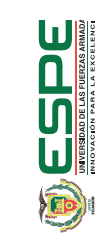


A partir de las entrevistas realizadas se comprendió desde una perspectiva personal la influencia de los cursos realizados, obteniendo que la mayoría de encuestados los consideró como positivos, debido a que, brindó oportunidades de crecimiento personal, recalcando que la metodología y teoría enseñada a los niños pudieron mejorar su destreza lógica matemática, solventar problemas, pensamiento creativo y acceso a información técnica, estableciendo el impacto integral a la vida de los niños y jóvenes participantes del proyecto.

Se consiguió el fomento de la creatividad, el emprendimiento y orientación vocacional enfocada hacia carreras tecnológicas o ingenierías. Lo cual fue demostrado en las encuestas obteniendo que el 71,4\% consideró que se aumentó el interés por dichas ramas de la ciencia. Teniendo en cuenta que a través de actividades lúdicas los niños pudieron construir su pensamiento lógico y tomando en cuenta que Isbell y Yoshizawa (2016) mencionan en su libro From Nuturing Creativity: An essencial mindset for young children's learning a cuatro habilidades y destrezas indispensables que necesitan los niños ahora y para el futuro, propuestas por expertos, líderes en educación y negocios, las cuales son las cuales son: pensamiento creativo, flexibilidad, resolución de problemas y generar ideas innovadoras.

Con la información recopilada es indudable el impacto social que tuvo la introducción del conocimiento y apropiación de nuevas tecnologías en edades iniciales, resultando beneficioso para el desarrollo cognitivo desde varias perspectivas. Una de ellas el pensamiento lógico-matemático y el fomento de habilidades para desenvolverse en la sociedad de la información. En la exposición final, supieron exponer todo el procedimiento para su elaboración y finalidad, enfocada en resolver un problema de la vida cotidiana. Se pudo evidenciar el proceso creativo de los niños, al construir y diseñar la interfaz y jugar con las variables. Además, aprendieron a planificar, controlar el tiempo y tomar decisiones para lograr su objetivo, es decir, se fortalecieron y potencializaron las funciones ejecutivas del lóbulo pre frontal; la creatividad, toma de decisiones, ejecución de actividades complejas, la inteligencia (Tirapu et al., 2002).

Se logró desarrollar su pensamiento lógico, puesto que los participantes, aprendieron a estructurar sus ideas para generar órdenes de programación e ingresarlas en la plataforma, siendo el niño capaz de entender y usar condiciones lógicas básicas. Dicho proceso es considerado como una construcción abstracta del pensamiento, anterior a materializarla a través de recursos. Los niños necesariamente requirieron iniciar un proceso de análisis para identificar las herramientas y elementos adecuados para construir su código y posteriormente programarlo. Consiguiendo crear simbolismos, conceptos y representaciones mentales que ayudaron a conformar ideas creativas para la elaboración de un producto.

Por otra parte, gracias a la aplicación de los talleres, se pudo sembrar la inquietud en los niños y adolescentes de ser desarrolladores de tecnología y no simples consumidores. Desde esta perspectiva, se respalda el inicio en edades infantiles a actividades que despierten la curiosidad e interés por brindar ideas innovadoras dentro de las ciencias técnicas.

Adicional a ello, este tipo de actividades que brindaron conexiones directas con ciencias técnicas y que están íntimamente relacionadas con la tecnología permiten potenciar la motivación, autonomía, desarrollo de distintas formas de comunicar sus ideas y mejorar sus habilidades sociales, reflexión, organización de ideas y trabajo en equipo; ya que existió la oportunidad de mantener contacto con otros niños y compartir sus ideas y trabajos, con el fin de buscar la mejora a través 
de un trabajo mancomunado de descubrimiento y experimentación. (Hong \& Trepanier-Street, 2004).

Para el acercamiento a la programación resulta viable el uso de Scratch y Arduino como herramientas didácticas. Gracias a su fácil acceso, bajo costo y presentación interactiva, para ser utilizada por niños y jóvenes como un acercamiento a ciencias técnicas. Motivando así, a continuar con el aprendizaje para a futuro ser ciudadanos que propongan nuevas tecnologías nacionales.

\section{REFERENCIAS}

Arribas, D. (2016). Scratch como recurso educativo para la enseñanza deprogramación en $3^{\circ}$ de ESO. Obtenido de Repositorio de la Universidad de Rioja: https://biblioteca.unirioja.es/tfe_e/TFE001417. pdf

Bailey, M. (1 de Agosto de 2018). Scratch Programming. Obtenido de Oregon State University: http://web.engr.oregonstate.edu/ mib/ scratch/scratch. 1 pp.pdf

Blanco, R. (23 de Septiembre de 2009). El pensamiento lógico desde la perspectiva de las neurociencias cognitivas. Oviedo, España.

Bourret, R. (s.f.). An introduction to programming with Scratch. Obtenido de RpBourret: http://www.rpbourret.com/scratch/BourretScratchProgramming.pdf

Hernández, Londoño, D. A. (2016). Interfaz de programación visual como herrramienta educativo para el desarrollo de competencia en ciencia y tecnología por parte de niños, jóvenes y educadores. Medellín.
Hernández, R., Fernández, C., \& Baptista, P. (2014). Metodología de la Investigación sexła edición. Mexico D.F: Mc Graw Hill.

Hong, S. B., \& Trepanier-Street, M. (2004). Technology: A Tool for Knowledge Construction in a Reggio Emilia Inspired Teacher Education Program. Early Childhood Education Journal, 87-94. Recuperado en: https://link.springer.com/article/10.1007/s10643-004-7971-z.

Ilvay, Taday, R. B. (Recuperado en: http://dspace.espoch.edu.ec/ bitstream/123456789/3276/1/108T0089.pdf de 2014). Sistema de Educación para niños de 3 a 5 años, mediante un robot controlado por el sensor KINECT. Riobamba, Chimborazo, Ecuador.

Isbell, R., \& Yoshizawa, S. (2016). From Nurturing Creativity: An Essential Mindset for Young Children's Learning. The National Association for the Education of Young Children.

Jiménez, N. (Recuperado en: https://e-archivo.uc3m.es/ bitstream/handle/10016/23818/TFG_Nuria_DeBenito_Jimenez. pdf? sequence $=1$ \&isAllowed $=y$ de 2015). Desarrollo de una plataforma didáctica para la educación infantil. Madrid, Universidad Carlos III de Madrid.

La Hora. (09 de Mayo de 2017). Casa Somos ofrece más de 2300 cursos gratuitos. La Hora, págs. https://lahora.com.ec/noticia/1 102056405/ casa-somos-ofrece-ms-de-2300-cursos-gratuitos-.

Marji, M. (2014). Learn to program with Scratch. San Francisco: Library of Congress Cataloging in Publication Data.

Municipio del Distrito Metropolitano de Quito. (2018). Casas Somos. Municipio del Distrito Metropolitano de Quito. Obtenido de 
Casas Somos: http://www.quito.gob.ec/index.php/municipio/66secretarias/277-casa-somos

Mustard , J. F. (2009). Early Human Development - Equity from the Start - Latin America. Revista Latinoamericana de Ciencias Sociale, Niñez y Juventud, 639-680. Recuperado en: http://www.scielo.org.co/ $\mathrm{pdf} / \mathrm{rlcs} / \mathrm{v} 7 \mathrm{n} 2 / \mathrm{v} 7 \mathrm{n} 2 \mathrm{a} 04 . \mathrm{pdf}$.

Ojeda, Carpio, B. E. (http://repositorio.utmachala.edu.ec/ bitstream/48000/7038/1/CD00067-2016-TESIS-COMPLETA.pdf de 2015). Tesis: Incidencia de la actividad lúdica como estrategia metodológica para el desarrollo del pensamiento lógico matemático en los niños y niñas del C.E.I. de "María Franco de Carrillo" y primer año de la escuela de educación básica "Galo Plaza Lasso"". Machala, El Oro, Ecuador.
Rubio, Centeno, R. (https://uvadoc.uva.es/bitstream/10324/1486/1/ TFG-B.109.pdf de 2012). El desarrollo lógico-matemático del niño a través de las tecnologías de la información y la comunicación. Segovia.

Tinajero, A. (2014). Trayectorias de Neurodesarrollo en el Ecuador: Una lectura de los indicadores de desarrollo humano. Guayaquil: http://docplayer.es/592891 16-Trayectorias-de-neurodesarrollo-enel-ecuador-una-lectura-de-los-indicadores-de-desarrollo-humanoalfredo-tinajero-febrero-2014-guayaquil.html.

Tirapu, Ustárroz, J., Muñoz, Céspedes, J. M., \& Pelegrín, Valero, C. (2002). Funciones ejecutivas: necesidad de una integración conceptual. Revista de Neurología, 673-685. Recuperado en: https://pdfs.semanticscholar. org/f6f1/b7ec7671afea34cb9cfae7804ee13c369368. pdf.

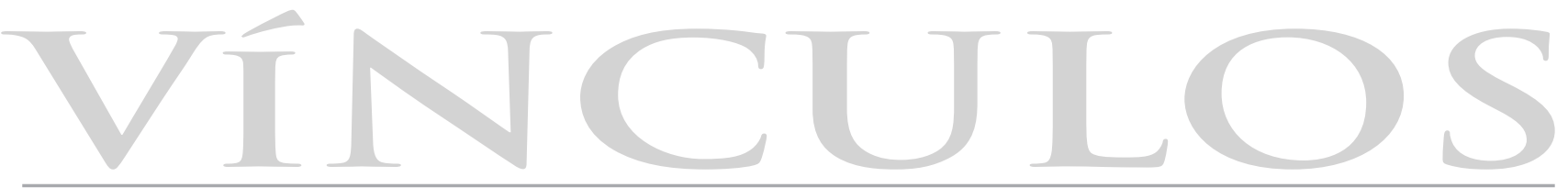

U N I V E R S D A D D E L A S F U ER Z A S A R M D A S E S P 